\title{
Enhanced isolation of influenza viruses in qualified cells improves the probability of well-matched vaccines
}

Heidi Peck $\mathbb{D}^{1,5 凶}$, Karen L. Laurie $\mathbb{i D}^{2,5 凶}$, Steve Rockman ${ }^{2,3}$, Vivian Leung ${ }^{1}$, Hilda Lau ${ }^{1}$, Sally Soppe ${ }^{1}$, Cleve Rynehart ${ }^{1}$, Chantal Baas ${ }^{2}$, Heidi Trusheim ${ }^{4}$ and lan G. Barr ${ }^{1,3}$

Influenza vaccines are utilised to combat seasonal and pandemic influenza. The key to influenza vaccination currently is the availability of candidate vaccine viruses (CVVs). Ideally, CVVs reflect the antigenic characteristics of the circulating virus, which may vary depending upon the isolation method. For traditional inactivated egg-based vaccines, CVVs are isolated in embryonated chicken eggs, while for cell-culture production, CVV's are isolated in either embryonated eggs or qualified cell lines. We compared isolation rates, growth characteristics, genetic stability and antigenicity of cell and egg CVV's derived from the same influenzapositive human clinical respiratory samples collected from 2008-2020. Influenza virus isolation rates in MDCK33016PF cells were twice that of eggs and mutations in the HA protein were common in egg CVVs but rare in cell CVVs. These results indicate that fully cell-based influenza vaccines will improve the choice, match and potentially the effectiveness, of seasonal influenza vaccines compared to egg-based vaccines.

npj Vaccines (2021)6:149; https://doi.org/10.1038/s41541-021-00415-3

\section{INTRODUCTION}

Influenza is a highly contagious, febrile respiratory illness that is responsible for an estimated 300,000-650,000 deaths annually ${ }^{1,2}$. Vaccination is the most effective treatment to prevent infection. For the majority of influenza vaccines that are currently produced, the supply of suitable influenza seed viruses for vaccine production depends on the isolation and propagation of influenza viruses from original clinical respiratory samples (OCS).

Traditionally, influenza vaccines have been produced by propagating the viruses required in embryonated chicken eggs. Clinical samples are directly inoculated into the amniotic or allantoic cavities of eggs ${ }^{3}$. Further manipulations on these initial virus isolates, such as by reassorting with laboratory-adapted viruses, are then required to generate high yielding viruses suitable for vaccine production ${ }^{4}$. Alternatively, the isolation and propagation of influenza viruses can be performed in continuous cell culture that has been qualified for use in human vaccines ${ }^{5}$. Concurrent to virus isolation for vaccine candidate production, influenza viruses are typically propagated in cell lines in surveillance laboratories to isolate viruses for antigenic analysis. Many continuous cell lines have been shown to support the growth of influenza viruses, such as Madin-Darby Canine Kidney $(\mathrm{MDCK})^{6-8}$, MDCK-SIAT1 (MDCK cells that overexpress $a-2,6-$ sialoglycans) ${ }^{9}$, MDCK-SIAT1-TMPRSS2 (MDCK-SIAT1 cells expressing transmembrane protease, serine 2$)^{10}, \mathrm{hCK}$ (MDCK cells that overexpress a-2,6-sialoglycans and have extremely low expression of $a-2,3$-sialoglycans) ${ }^{11}$, African green monkey kidney (Vero) ${ }^{12,13}$, baby hamster kidney (BHK-21) ${ }^{6}$, SJPL (St Jude porcine lung cells) ${ }^{14}$, Lewis lung carcinoma monkey kidney (LLC-MK2) ${ }^{7}$, swine nasal epithelial cells (siNEC) and tracheal epithelial cells (siTEC) ${ }^{15}$ and EB66 ${ }^{{ }^{\oplus} 16}$ cells. Among these, MDCK and MDCK-SIAT1 cell lines are commonly used as they are highly permissive to influenza viruses, easy to cultivate and progeny virus contain few modifications from the $\mathrm{OCS}^{9}$. Currently, the majority of influenza virus vaccines are still manufactured in embryonated hen's eggs ${ }^{17,18}$, although alternative non-egg host systems, such as the use of mammalian cells and recombinant protein vaccines have been licensed (reviewed in ref. ${ }^{5}$ ).

It is well recognised that the propagation of influenza viruses in eggs can result in the selection of egg-adapted viruses with mutations in the influenza virus surface protein, haemagglutinin $(\mathrm{HA})^{19-21}$. Mutations in the antigenic sites of the HA protein may alter the immune epitopes of the virus and alter the antigenicity of the virus ${ }^{21-25}$. This has the potential to reduce the effectiveness of the influenza vaccine ${ }^{22,26,27}$. In the past eleven years, $A(H 3 N 2)$ viruses have antigenically drifted rapidly resulting in more frequent updates to the recommendation for the $A(H 3 N 2)$ component of the vaccine compared to $A(\mathrm{H} 1 \mathrm{~N} 1)$ pdm09 and influenza B (Victoria or Yamagata) ${ }^{28}$. Egg adaptation of viruses occurs in both influenza $A$ and $B$ viruses, but are most common for $\mathrm{A}(\mathrm{H} 3 \mathrm{~N} 2)$ viruses $^{29}$ and are in part responsible for the reduced efficacy of this vaccine component ${ }^{22,26,27}$. Thus, the challenge to isolate $\mathrm{A}(\mathrm{H} 3 \mathrm{~N} 2)$ candidate vaccine viruses (CVV's) in eggs that are antigenically well matched to circulating strains and do not contain undesirable egg adaptations in the HA protein is still ongoing.

The aim of this study was to assess the isolation rates, genetic and antigenic characteristics and growth properties of influenza viruses isolated in a proprietary MDCK cell line, MDCK33016PF ${ }^{30}$, compared to embryonated hens eggs, for potential use as influenza CVV's. MDCK33016PF cells are highly permissive to influenza viruses whilst having limited ability to propagate other human viruses, a characteristic similar to embryonated hen's eggs $^{31-33}$. It was hypothesised that propagation of human influenza viruses in mammalian cells would result in improved isolation of viruses with fewer mutations in the HA compared to isolation in embryonated hen's eggs.

\footnotetext{
${ }^{1}$ WHO Collaborating Centre for Reference and Research on Influenza, VIDRL, The Peter Doherty Institute for Infection and Immunity, Parkville, VIC, Australia. ${ }^{2}$ Seqirus Ltd, Parkville, VIC, Australia. ${ }^{3}$ Department of Immunology and Microbiology, The University of Melbourne, Parkville, VIC, Australia. ${ }^{4}$ IDT Biologika GmbH, Dessau, Germany. ${ }^{5}$ These authors contributed equally: Heidi Peck, Karen L. Laurie. ${ }^{凶}$ email: Heidi.Peck@influenzacentre.org; Karen.Laurie@seqirus.com
} 


\section{RESULTS}

Higher isolation rates of human seasonal influenza viruses in MDCK33016PF cells compared to embryonated hen's eggs

The isolation rate of human viruses from OCS was directly compared between cells and eggs from 2008-2020. OCS from 895 individuals were inoculated into both substrates and were assessed for virus growth by Haemagglutination (HA) assay. Overall, 336 samples (37.5\%) were isolated in both cells and eggs, 389 samples (43.5\%) were isolated in cells only, 27 samples were isolated in eggs only (3\%) and $143(16 \%)$ samples were not isolated in either substrate (Fig. 1a). Samples were twice as likely to be isolated in cells $(81.0 \%)$ than eggs $(40.6 \%)(p<0.00001)$.

For all influenza A subtypes and B virus lineages, a higher proportion of viruses were isolated in cells than in eggs (Fig. 1a). Over $80 \%$ of viruses from each subtype/lineage were isolated in cells (range $74.8-82.6 \%$ ) (Fig. 1a). Almost half of $\mathrm{A}(\mathrm{H} 1 \mathrm{~N} 1)$ pdm09 viruses were isolated in eggs (48.7\%), whilst lower proportions of $\mathrm{A}(\mathrm{H} 3 \mathrm{~N} 2) \quad(37.7 \%), \mathrm{B} /$ Victoria (37.9\%) and B/Yamagata (33.3\%) viruses were isolated in eggs (Fig. 1a).

During this study, an additional 369 OCS were inoculated into cells only giving an overall isolation rate in cells for all subtypes/ lineages of $78.5 \%$ (range $73.5-80.4 \%$, Fig. 1b).

Analysis by sample year and subtype/lineage showed that isolation of viruses in cells was more consistent than eggs (Fig. 2). There was significantly less variation in the isolation rate of viruses in cells as compared to eggs, from year to year, for $\mathrm{A}(\mathrm{H} 1 \mathrm{~N} 1) \mathrm{pdm} 09$ $(p=0.0153)$ and $\mathrm{B} /$ Yamagata $(p=0.0462)$ viruses. There was no statistically significant difference in variation in the isolation rate of viruses in cells as compared to eggs, from year to year for A

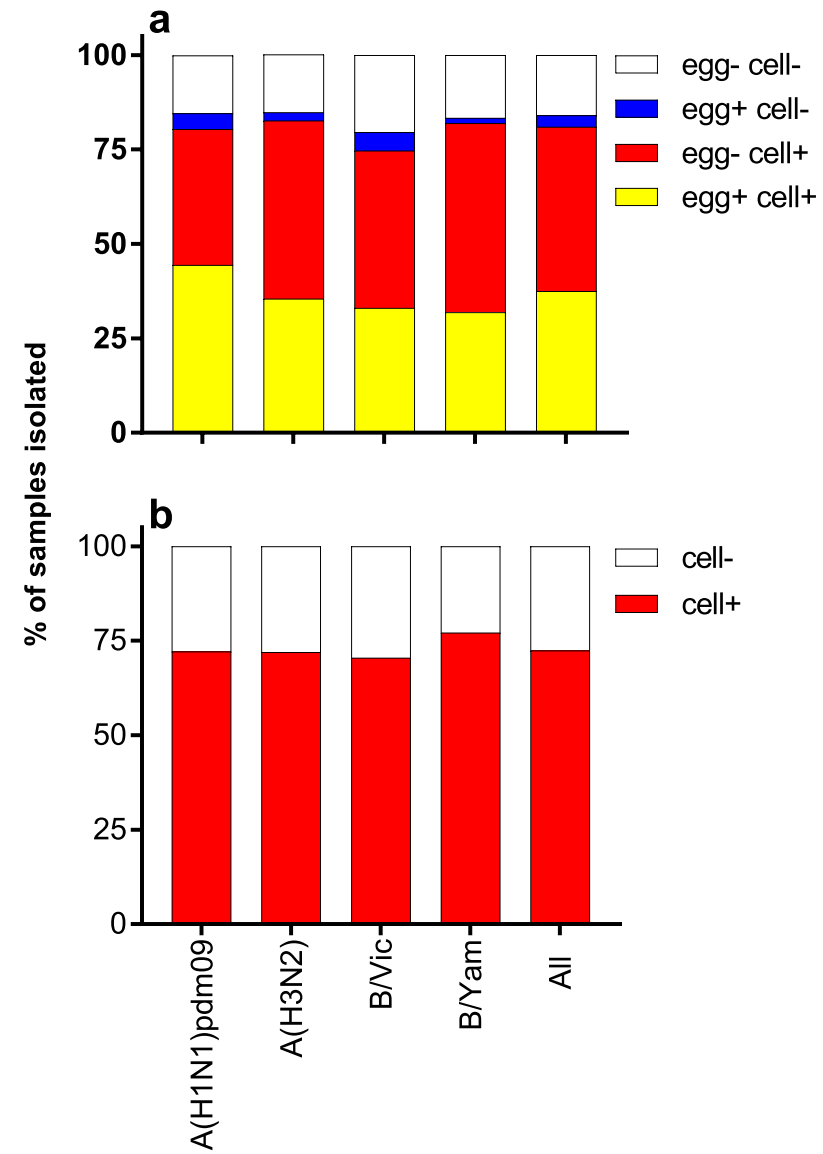

Fig. 1 The proportion recovery in each culture type of viruses grouped by subtype/lineage and of all samples combined. a 895 samples were inoculated into both cells and eggs and b 369 samples were inoculated into cells only.
(H3N2) $(p=0.0600)$ and B/Vic viruses $(p=0.0961)$. Early in the study, the isolation rates were comparable between cells and eggs, but very low isolation rates in eggs (less than 50\%) were observed between 2011-2013, and in 2015 for $A(H 1 N 1) p d m 09$ viruses, for 2011-2017, 2020 for $A(H 3 N 2)$ and 2011-2015 for B/Victoria and for 2010-2014 for B/Yamagata viruses (Fig. 2, see Supplementary Table 1 for raw data).

In the first few years after the 2009 pandemic, isolation of A (H1N1)pdm09 viruses in embryonated eggs by allantoic inoculation of influenza-positive OCS was quite successful. However, by $2012, \mathrm{~A}(\mathrm{H} 1 \mathrm{~N} 1)$ pdm09 isolation rates had fallen to around $20 \%$ and the inoculation method for $\mathrm{A}(\mathrm{H} 1 \mathrm{~N} 1)$ pdm09 OCS reverted to the methods used prior to 2009 for seasonal $A(\mathrm{H} 1 \mathrm{~N} 1)$ viruses. They consisted of initial isolation in the amniotic cavity followed by passaging of viruses in the allantoic cavity of embryonated hen's eggs. From 2013, egg isolation rates for A(H1N1)pdm09 containing samples improved and by 2017 had reached $75 \%$ or greater with some years having higher isolation rates in eggs than in cells (Fig. 2a), although only a small number of isolations were attempted in eggs in these years (isolations for 2018: eggs- 17/ 17 , cells $-41 / 44$; 2019: eggs $-4 / 4$, cells $-31 / 48$; 2020: eggs $-2 / 2$, cells - 11/18).

The genetic and antigenic complexity of $\mathrm{A}(\mathrm{H} 3 \mathrm{~N} 2)$ has increased in recent years resulting in the designation of multiple genetic clades and sub-clades based on the HA gene ${ }^{34}$. The isolation rate was assessed by clade for $\mathrm{A}(\mathrm{H} 3 \mathrm{~N} 2)$ viruses where sequences were available (Fig. 3). At least one virus was isolated in eggs and in cells for each clade, except for $3 \mathrm{c} 2 \mathrm{a} 1 \mathrm{~b} / 131 \mathrm{~K} / 186 \mathrm{D}$, where only one OCS was received and only grew in eggs. Viruses in the $3 a, 3 b$ and $3 c$ clades, which circulated in 2011-2013, were isolated in very high proportions (95-100\%) in cells but only at low proportions $(20-24 \%)$ in eggs. Similarly, two-thirds of viruses in the 3c.2a and 3c.2a1 clades, which circulated in 2014-2017, were isolated only in cells. This is in contrast to viruses early in the study (2009-2010), or more recently (2018-2020), which have been more readily isolated in both cells and eggs.

\section{Differential binding of turkey and guinea pig red blood cells} to virus isolates over time

Chicken, turkey and mammalian red blood cells (RBC) have traditionally been used to detect and quantitate influenza viruses for several decades. RBC from different species express different levels of glycan linkages making them useful to assess changes in the HA receptor binding preferences ${ }^{24}$. Guinea pig (GP) RBC express more a2,6-linked sialylated N-glycans than turkey RBC and chicken RBC, which have more a2,3-linked sialylated N-glycans ${ }^{35}$. Throughout this study the titre of cell-grown viruses was measured by HA assay using GP and turkey RBC, effectively assessing the binding preferences of HA over time. Cell-derived $A$ (H1N1)pdm09 viruses are consistently bound to both GP and turkey RBC with titres increasing from 2011 to 2019 following the introduction of the pandemic $A(\mathrm{H} 1 \mathrm{~N} 1)$ virus in 2009 (Fig. 4b, c). Increases in $\mathrm{HA}$ titres for $\mathrm{A}(\mathrm{H} 1 \mathrm{~N} 1)$ pdm09 viruses grown in eggs was also observed (Fig. 4d) from 2014. The proportion of MDCK33016PF cells infected with a virus, as determined by flow cytometry detecting nucleoprotein (NP) expression as a marker of virus infection, was greater than $50 \%$ for all years (Fig. $4 \mathrm{a}$ ). The majority of $A(H 3 N 2)$ viruses (87\%) lost the ability to bind to turkey RBC from 2011-2018 (Fig. 4g) yet retained binding to GP RBC (Fig. 4f). A consistent level of infection in cells was maintained (Fig. 4e) and consistent HA titres were observed in egg-grown viruses as measured with GP RBC (Fig. 4h). These data indicate a change in the binding preference of the H3 HA away from avianlike receptors towards mammalian-like receptors during the study period. HA titres for cell-grown influenza B viruses were consistent over time (see Supplementary Information, Supplementary Fig. 1). 

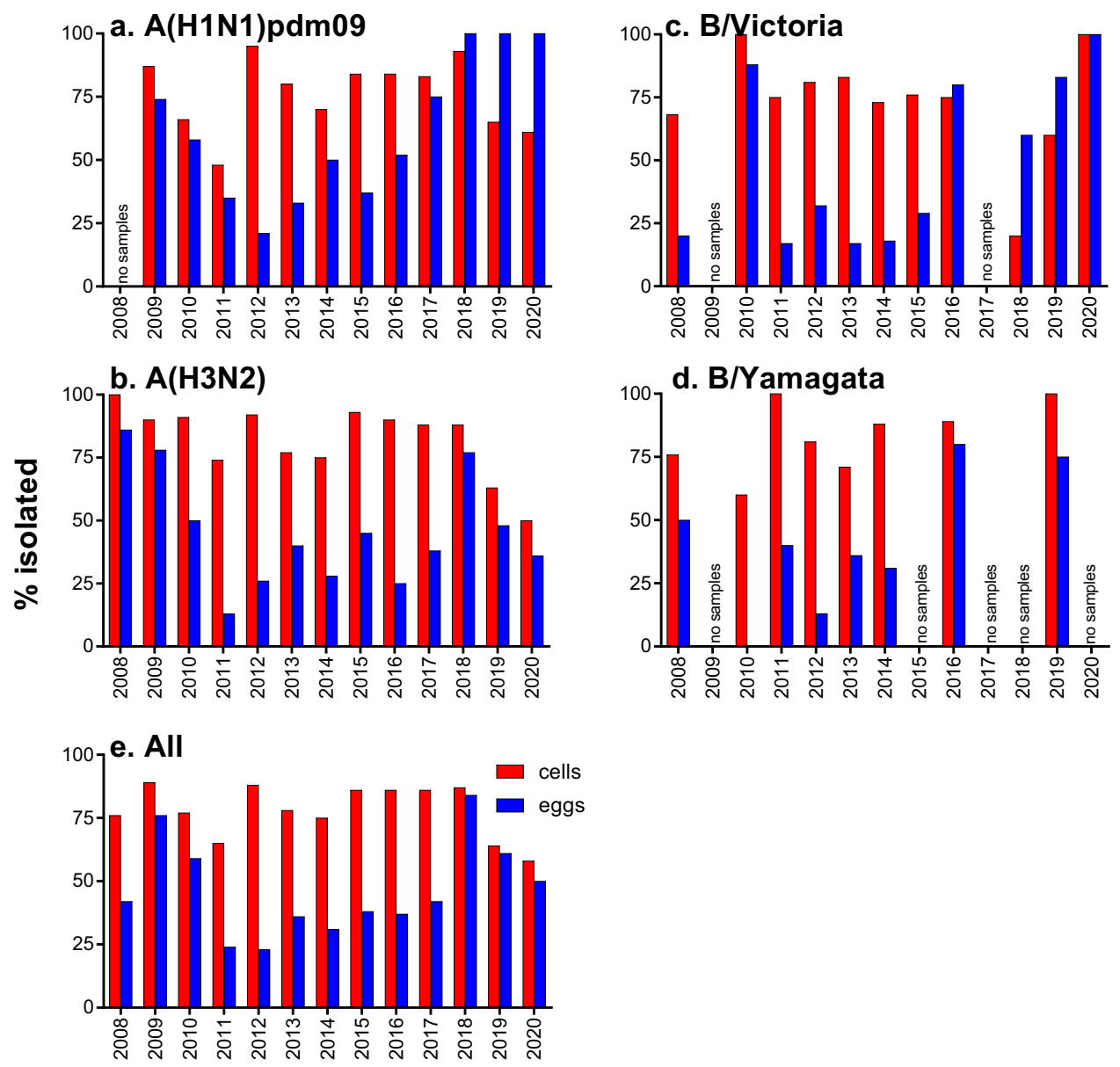

Fig. 2 Isolation rates of all human influenza viruses tested in cells and eggs. Proportion recovered in cells (red bar) or eggs (blue bar) by year of sample collection is shown for $\mathrm{A}(\mathrm{H} 1 \mathrm{~N} 1)$ pdm09 (a), $\mathrm{A}(\mathrm{H} 3 \mathrm{~N} 2)(\mathbf{b}), \mathrm{B} /$ Victoria (c), B/Yamagata (d) and all (e) viruses.

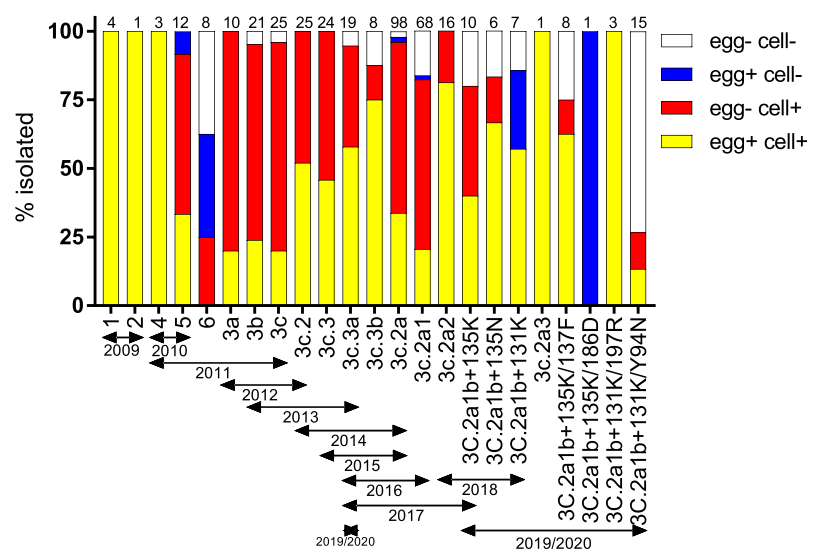

Fig. 3 Isolate rates of $A(H 3 N 2)$ viruses tested in cells and eggs by clade. Numbers at the top of the bars indicate the number of viruses attempted in each clade. Virus clades were determined by sequencing the HA gene.

\section{Comparison of virus growth for cell and egg isolates}

The HA titres of viruses that grew in cells only, eggs only or both cells and eggs were compared. Influenza A viruses that were isolated in both cells and eggs had higher HA titres in cells than influenza $A$ viruses that grew in cells only (Fig. 5a, b). The amount of viral RNA (as determined by real-time PCR cycle threshold values) was also higher in the OCS of $A(\mathrm{H} 1 \mathrm{~N} 1)$ pdm09 samples that were isolated both in cells and eggs as compared to $A(\mathrm{H} 1 \mathrm{~N} 1)$ pdm09 samples isolated in cells alone (Fig. 5c). These data indicate that the OCS's containing higher amounts of virus were more often isolated in both cells and eggs whilst samples containing less virus were more likely to be isolated only in cells. Influenza A viruses that were isolated in both cells and eggs had similar HA titres in eggs as influenza A viruses that grew in eggs only (Fig. 5a, b). There was no statistical difference in titre for influenza $B$ viruses that were isolated in cells alone compared to those isolated in both cells and eggs (Fig. 5d).

\section{Comparison of mutations in the HA and NA genes in the cell and egg isolates}

A subset of viruses $(n=68)$ was assessed for the stability of the HA sequence after isolation and subsequent passage in both eggs and cells by consensus Sanger sequencing of the HA gene. Nonsynonymous mutations in the HA gene were observed in 93\% (63/ 68) of viruses passaged in eggs and $10 \%(7 / 68)$ of viruses passaged in cells, as compared to the HA gene sequence of the OCS (Fig. 6). The HA sequences were assessed for a further 98 viruses passaged in cells only, and non-synonymous mutations in the HA were observed in $11 \%(11 / 98)$ of viruses; taken together, mutations were observed in $11 \%(18 / 166)$ of all sequenced viruses passaged in cells (Fig. 6). Of the seven viruses with mutations observed in both cells and eggs, common mutations following cell- and egg-passage were observed in four of the viruses (see Supplementary Information, Supplementary Table 2). Of the four viruses, three had polymorphisms in the HA of the OCS, that when isolated selected the same amino acid in both cells and eggs, indicating that there was no selection by the isolation substrate 

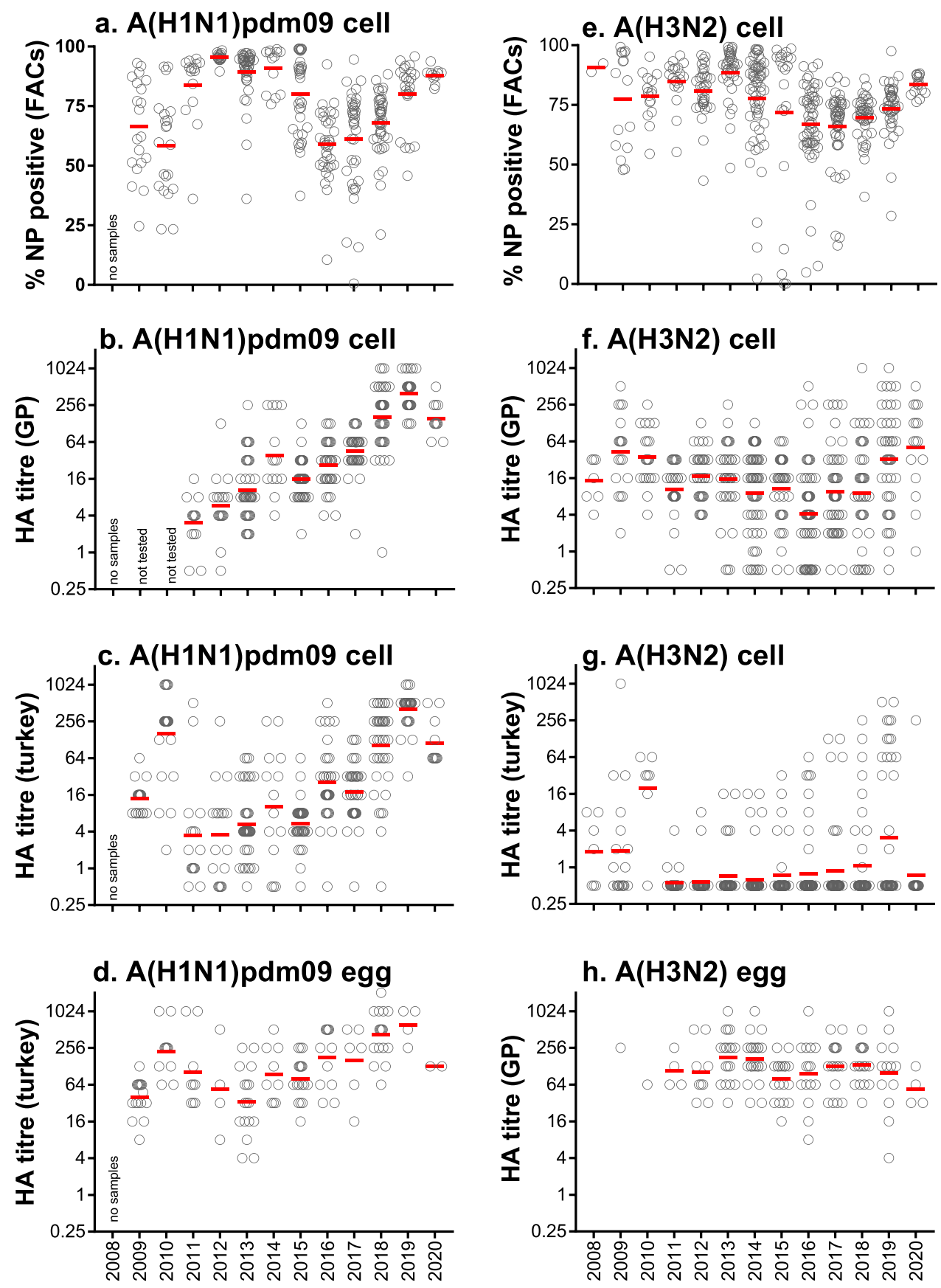

\section{sample year}

Fig. 4 HA titres of influenza $\mathbf{A}$ isolates to GP and turkey RBC, from 2008-2020. a, e Cell-grown $A(H 1 N 1) p d m 09$ and $A(H 3 N 2)$ viruses were assessed for infectivity of cells by flow cytometry. The red bar indicates the mean. HA titres of cell-grown isolates to GP RBC (b, $\mathbf{f})$ and turkey $\mathrm{RBC}(\mathbf{c}, \mathbf{g})$. d The HA titre of egg-grown $\mathrm{A}(\mathrm{H} 1 \mathrm{~N} 1)$ pdm09 viruses was measured using turkey RBC. $\mathbf{h}$ The HA titre of egg-grown $\mathrm{A}(\mathrm{H} 3 \mathrm{~N} 2)$ viruses was measured using GP RBC. (b-d, f-h) Red bar indicates geometric mean titre).

(see Supplementary Information, Supplementary Table 2). Following passage in eggs, the proportion of viruses with mutations was similar for both influenza $A$ subtypes and influenza $B$ viruses ( $A$ (H1N1)pdm09-86\% (24/28), A(H3N2)-100\% (26/26), B-93\% $(13 / 14))$. Influenza $B$ viruses were less likely to acquire mutations in the HA following passage in cells $(3 \% 1 / 36)$, than influenza $A$ viruses $(A(\mathrm{H} 1 \mathrm{~N} 1)$ pdm09-11\% $\quad(7 / 66), \quad A(\mathrm{H} 3 \mathrm{~N} 2)-16 \% \quad(10 / 64)$ (Fig. 6). The NA gene sequences were also assessed by consensus Sanger sequencing. Adaptive mutations in the NA gene of egggrown viruses was less common, observed in $40 \%(6 / 15)$ of $A$ (H1N1)pdm09 viruses, $8 \%(2 / 26)$ of $A(H 3 N 2)$ viruses and no $(0 / 9)$ influenza B viruses. Adaptive mutations in the NA gene of cellgrown viruses was rare, observed only in $7 \%(1 / 15)$ of $A(\mathrm{H} 1 \mathrm{~N} 1)$ pdm09 viruses and none in $A(H 3 N 2)(0 / 25)$ or influenza $B(0 / 9)$ viruses (Supplementary Information, Supplementary Table 2).

Mutations were located mainly in the head of the HA protein in both egg and cell passaged viruses (Fig. 6 and Supplementary Information, Supplementary Table 2). Over twice as many mutation sites were located in antigenic sites for $A(H 3 N 2)$ viruses passaged in eggs $(65 \% 13 / 20)$ than passaged in cells $(30 \% 3 / 10)$ (Fig. 6b), and for influenza B viruses (eggs 100\% 3/3, cells 0\% 0/2)(Fig. 6c). Similar proportions of mutation sites were located in the 

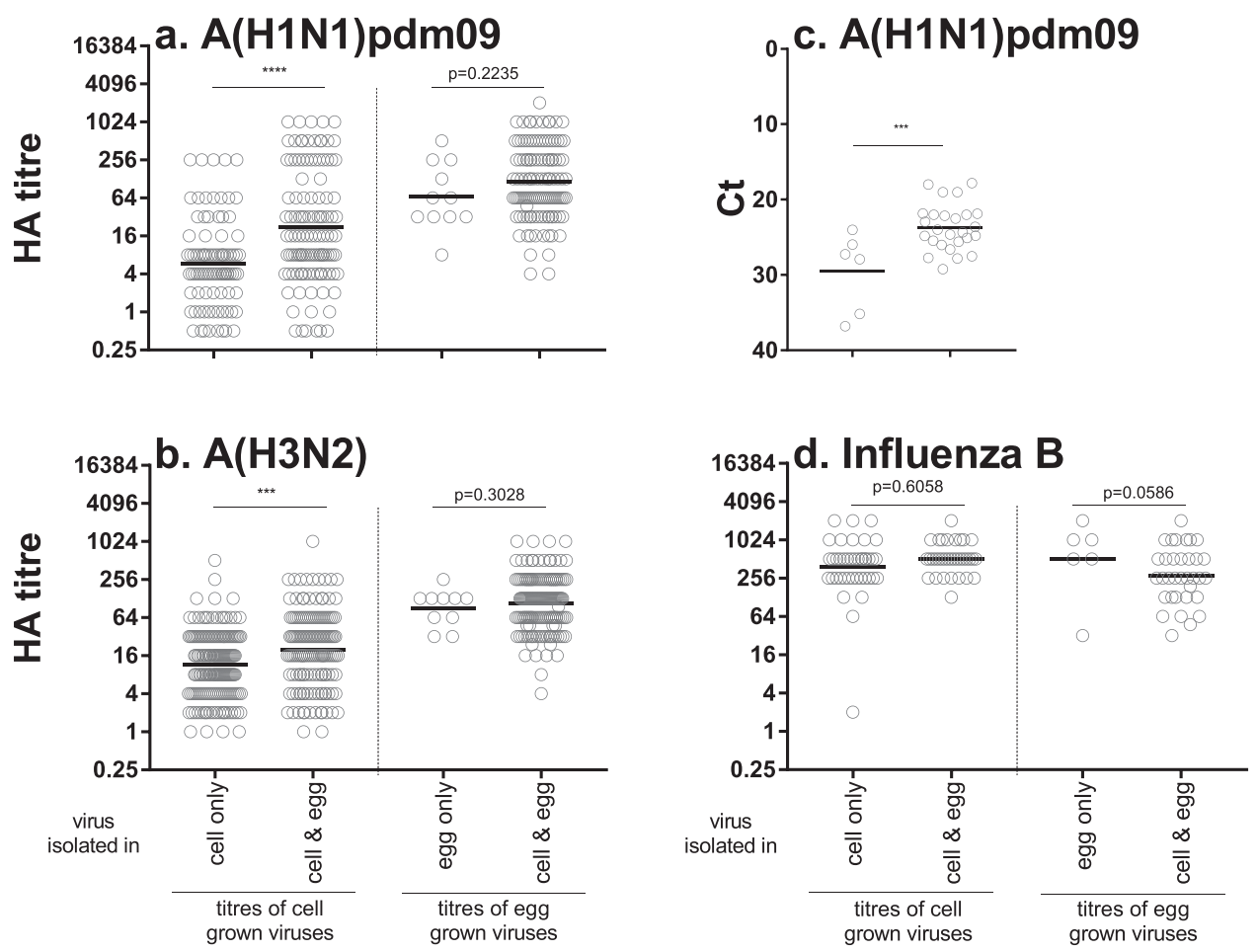

Fig. 5 Assessment of HA titre for cell-grown or egg-grown viruses. Viruses were inoculated into both cells and eggs and growth was assessed, as per Fig. 1a. Cell-grown viruses were grouped by viruses that were isolated only in cells as compared to viruses that were isolated in cells and eggs. Egg-grown viruses were grouped by viruses that were isolated only in eggs as compared to viruses that were isolated in cells and eggs. The HA titres were plotted for $\mathrm{A}(\mathrm{H} 1 \mathrm{~N} 1)$ pdm09 (a) and influenza $\mathrm{B}$ (d) as detected by turkey RBC and $\mathrm{A}(\mathrm{H} 3 \mathrm{~N} 2)(\mathbf{b})$ as detected by $\mathrm{GP}$ RBC. c The $\mathrm{Ct}$ of vRNA for a subset of $\mathrm{A}(\mathrm{H} 1 \mathrm{~N} 1)$ pdm09 samples was measured. ${ }^{* *} p<0.001,{ }^{* * *} p<0.0001$. The difference in $\mathrm{HA}$ titres or $\mathrm{Ct}$ was assessed using an unpaired $t$-test.

antigenic sites of $\mathrm{H} 1 \mathrm{HA}$ for egg (23.5\% 4/17) and cell (25\% 2/8) passaged viruses (Fig. 6a). One-quarter of mutations observed following passage in cells and in eggs resulted in mixed amino acids in the HA protein (Fig. 6).

Half the amino acid sites that mutated following passage in eggs (19/38) were shared among more than one virus (Fig. 6). Seven mutations were seen in at least four influenza $A$ viruses each and are indicative of common egg-adaptation mutations (S183P, L191I and Q223R in A(H1N1)pdm09 viruses, T160K, G186V, L194Pand S219Y/F in A(H3N2) viruses) $20,22,24,29,36$ (Fig. 6a, b). Mutations observed following passage in cells were only found in one or two viruses (Fig. 6a, b). The N196D/Y/X mutations in B/ Yamagata viruses is structurally equivalent to the N197S/X mutation in B/Victoria viruses and was observed in nine eggpassaged influenza B viruses (Fig. 6c).

Overall, these data indicate that cell-grown viruses are more representative of currently circulating strains in humans than egggrown influenza viruses.

\section{Egg adaptations in the HA protein affect the antigenicity of} egg-passaged viruses

The impact of egg adaptation/s in the HA protein on the antigenicity of $A(H 3 N 2)$ viruses was assessed by the haemagglutination inhibition $(\mathrm{HI})$ assay against a panel of post-influenza vaccination human sera. The reactivity of sera from patients that had received the egg-grown influenza virus vaccine was tested against cell- and egg-grown pairs of viruses from different $A$ (H3N2) genetic clades that circulated from 2014-2017.

Antibody responses expressed as geometric mean titres to egggrown viruses were significantly higher than antibody responses to the corresponding cell-grown viruses for all clades tested (Fig. 7a). These data indicate that sera obtained from postvaccination donors inhibited egg-grown viruses well, but did not inhibit cell-grown viruses well. The egg-grown viruses had all acquired T160K and L194P adaptations in the HA protein, which corresponded to adaptations in the egg vaccine received by the recipients (Fig. 7b). The T160K and L194P mutations are located in antigenic site B of the H3 HA protein. Thus it is likely that the postvaccine antibody response was directed to the egg adaptations and may not protect as well against infection with circulating viruses, which do not contain these adaptions in the HA protein.

\section{DISCUSSION}

This study demonstrated that human influenza viruses were more readily isolated in the qualified mammalian cell line, MDCK33016PF, than in embryonated hen's eggs. All subtypes and lineages were isolated at higher levels in MDCK33016PF cells and this remained consistent over the study period (2008-2020), while the isolation rate of viruses in embryonated eggs varied for each subtype and lineage. Changes in receptor binding preferences may influence the isolation of human influenza viruses, especially $A(H 3 N 2)$ viruses, in eggs ${ }^{24,37}$. Viruses that were isolated in the MDCK33016PF cell line had few mutations in the HA, whilst egg adaptation of the HA was common. The egg HA adaptations affected the antigenicity of the virus and reduced the recognition of vaccine-induced antibodies to circulating virus strains as demonstrated in this study and others ${ }^{38-40}$.

The difference in the isolation rate between cells and eggs is significant. Overall, for every five viruses inoculated into cells, on average four will grow, whilst for every five viruses inoculated into eggs, on average only two will grow. Thus at least twice as many inoculations into eggs compared to cells, are required to isolate the same number of viruses. This increased isolation rate in cells is directly related to the susceptibility of each substrate to influenza virus infection with human viruses having to undergo adaption to 

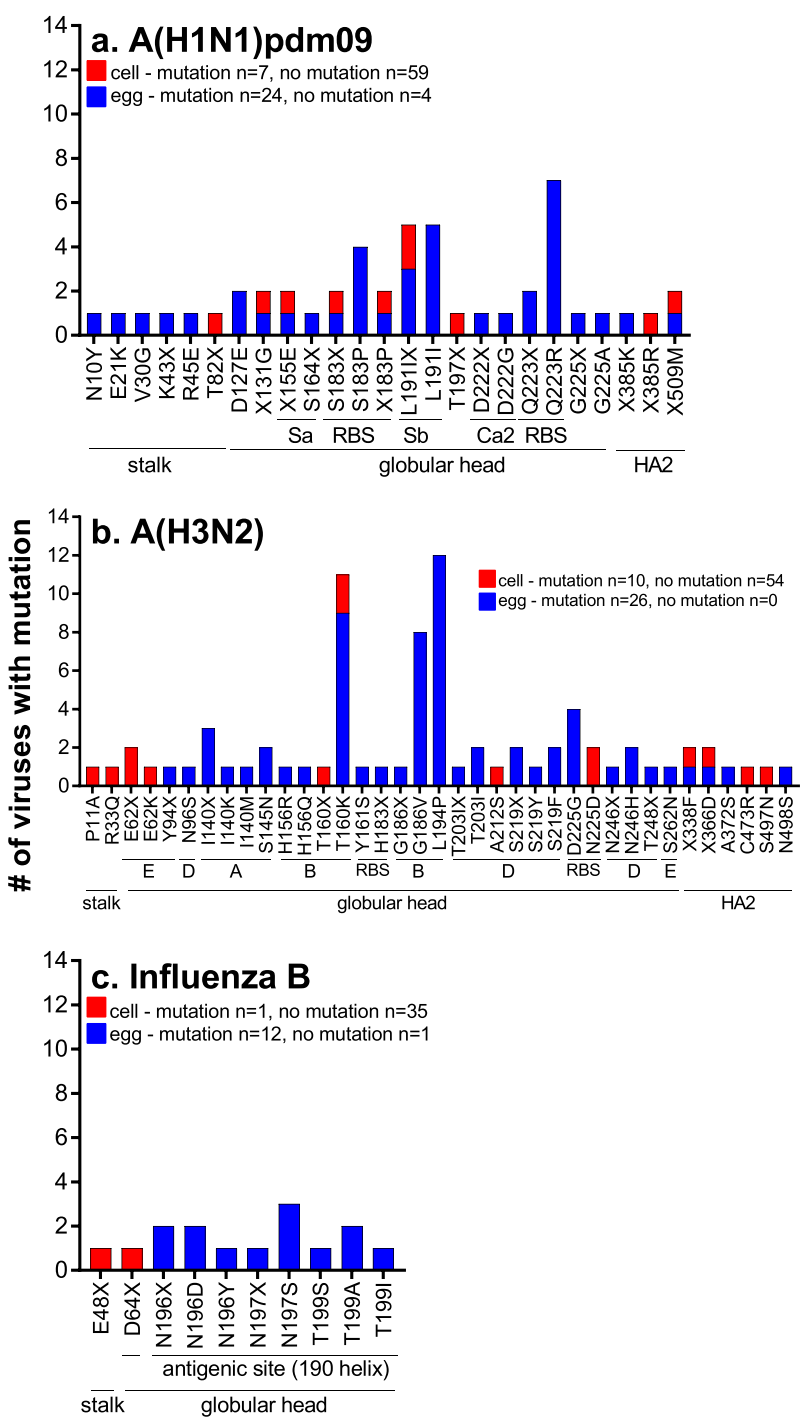

\section{mutation in HA protein}

Fig. 6 Assessment of mutations in the HA protein following virus isolation. The frequency of mutations in $\mathrm{HA}$ protein of viruses isolated in cells and eggs for $\mathrm{A}(\mathrm{H} 1 \mathrm{~N} 1) \operatorname{pdm09}(\mathbf{a}), \mathrm{A}(\mathrm{H} 3 \mathrm{~N} 2)$ (b), B/ Victoria and B/Yamagata (c) as compared to the OCS. The total number of cell and egg virus isolates with HA gene sequenced and the number of viruses with and without any mutation in the HA is indicated on each graph. The location of each mutation in the $\mathrm{HA}$ protein is indicated below the $x$-axis, as determined using ${ }^{26,58-60}$.

the avian (egg) host in order to replicate efficiently. Limited isolation of viruses in eggs reduces the choice and availability of viruses for influenza virus reassorting and vaccine manufacture. Poor yielding egg-derived viruses may also lead to a delay in vaccine availability due to the failure to have a high-yielding CVV available. The significantly improved isolation rates in MDCK33016PF cells increase the number of CVVs available for manufacture, and this increases the likelihood that a good growing, high yielding CVV will be available. Furthermore, the reduced number of mutations of cell CVVs compared to egggrown CVVs indicates that cell-derived isolation results in a bettermatched CVV to the circulating influenza viruses, especially with the $A(H 3 N 2)$ subtype.

The improved isolation rate of cells versus eggs may be explained by the level of sialylated $\mathrm{N}$-glycan expression. Human influenza viruses bind to sialylated $\mathrm{N}$-glycans and phosphorylated a

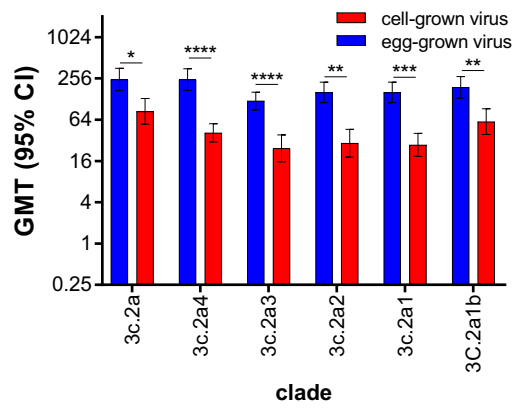

b

\begin{tabular}{|c|c|c|c|c|c|c|c|c|c|}
\hline \multirow{3}{*}{ Clade } & \multirow{3}{*}{ Virus } & \multirow{3}{*}{ passage } & \multicolumn{7}{|c|}{ HA position in MDCK 33016PF sequence } \\
\hline & & & 96 & 160 & 186 & 190 & 194 & 203 & 225 \\
\hline & & & $N$ & $T$ & G & $\mathrm{D}$ & L & $T$ & $\mathrm{D}$ \\
\hline \multirow[t]{2}{*}{ 3c.2a } & $\begin{array}{l}\text { A/Hong Kong/4801/2014 } \\
\text { (vaccine) }\end{array}$ & E4 & S & K & - & - & $P$ & & - \\
\hline & A/Michigan/15/2014 & P2 & - & - & - & - & - & & - \\
\hline \multirow{2}{*}{ 3c.2a4 } & A/Brisbane/32/2017 & E4 & - & K & - & - & $\mathrm{P}$ & & - \\
\hline & A/Brisbane/32/2017 & P2 & - & - & - & - & - & & - \\
\hline \multirow{2}{*}{$3 c .2 \mathrm{a3}$} & A/Singapore/TT1374/2016 & E6 & - & $\mathrm{K} / \mathrm{I}$ & $\mathrm{G} / \mathrm{V}$ & $\mathrm{G} / \mathrm{A}$ & $\mathrm{P} / \mathrm{L}$ & & G/D \\
\hline & A/Singapore/TT1374/2016 & P2 & - & - & - & - & - & & - \\
\hline \multirow{2}{*}{$3 c .2 \mathrm{a} 2$} & A/Brisbane/321/2016 & E6 & - & K & - & - & $P$ & & - \\
\hline & A/Brisbane/321/2016 & P2 & - & - & - & - & - & & - \\
\hline \multirow{2}{*}{ 3c.2a1 } & $\begin{array}{c}\text { A/Singapore/INFIMH-16- } \\
0019 / 2016\end{array}$ & E6 & - & K & - & - & $P$ & & - \\
\hline & $\begin{array}{c}\text { A/Singapore/INFIMH-16- } \\
0019 / 2016\end{array}$ & P2 & - & - & - & - & - & & - \\
\hline \multirow{2}{*}{ 3c.2a1b } & A/Victoria/624/2017 & E4 & & $\mathrm{X} 160 \mathrm{~T}$ & & & $P$ & $x$ & \\
\hline & A/Victoria/624/2017 & p2 & & & & & & & \\
\hline
\end{tabular}

Fig. 7 Assessment of impact of egg adaptation on antigenicity. a Analysis of post-vaccination serological responses to egg- and cellgrown virus pairs. Twenty human post-vaccination sera were tested by $\mathrm{HI}$ assay against the indicated virus. The Geometric Mean Titre (GMT) and 95\% confidence interval was calculated for each virus assessed. b Amino acid mutations in the HA-1 sequence, as compared to the cell sequence is shown for each virus included in (a). ${ }^{*} p<0.05,{ }^{* *} p<0.01,{ }^{* * *} p<0.001,{ }^{* * * *} p<0.0001$. The difference in GMT was assessed using a multiple $t$-test.

non-sialylated $\mathrm{N}$-glycans expressed on the human respiratory tract ${ }^{41-44}$. Studies using the Sambucus nigra (SNA) and Maackia amurensis (MAA) lectins show that human respiratory tissues express a2,6-linked sialylated $\mathrm{N}$-glycans more abundantly than a2,3-linked sialylated $\mathrm{N}$-glycans on cells, though both linkage types are present ${ }^{42,43}$. Similar patterns were observed on MDCK cells ${ }^{45}$. Studies of avian tissues show expression of both types of receptors, with an increased level of a-2,3-linked sialylated glycans in tissues ${ }^{46,47}$ and in the chorioallantoic and amniotic membranes in embryonated chicken eggs ${ }^{48}$. An increased expression of human-like surface receptors (a2,6-linked sialylated N-glycans) on MDCK33016PF cells as compared to eggs may explain their improved isolation rates of human influenza viruses. Increased expression of human-like surface receptors may lead to an increase in the avidity of the interaction between the viral HA and its receptor, improving isolation of samples with low amounts of virus or low HA affinity for its receptor ${ }^{9}$. In fact, we showed that influenza A samples isolated in cells only had lower viral loads in the OCS than influenza A samples that could be isolated in both cells and eggs. Over the course of the study, $A(H 3 N 2)$ viruses lost their ability to agglutinate turkey RBC and this directly correlated with a reduction of isolation of viruses in eggs, but not in cells. Others have also documented the loss of turkey RBC agglutination of $A(H 3 N 2)$ viruses over time and the accompanying reduction in $\mathrm{HA}$ affinity and specificity for its receptor ${ }^{24,49-51}$. Taken together, these data indicate that MDCK33016PF cells support the isolation 
of samples by increasing the avidity of the viral HA and the receptor on the cell surface.

This study demonstrated that viruses that are isolated in the MDCK33016PF cell line have fewer mutations in the HA gene, whilst egg adaptation mutations in the HA gene are common. Not unexpectedly, the majority of mutations occurred around the globular head of the HA, the region involved in virus binding to its cell receptor. For cell isolations of influenza viruses, $25 \%$ of mutations were located in antigenic sites, and $35 \%$ of mutations were located in antigenic sites or the RBS. Mutations were spread over the whole $\mathrm{HA}$, consistent with random mutation due to a lack of proof-reading ability in the polymerase of influenza virus ${ }^{52}$. In contrast, for egg-derived influenza virus isolates, mutations were predominantly in the antigenic sites and RBS (63\%). Mutations were particularly focused in these regions for the $\mathrm{H} 3 \mathrm{HA}(80 \%$ of mutations) and influenza B HA of egg-grown viruses (100\% of mutations). It is well known that egg adaptations in $A(H 3 N 2)$ viruses can affect antigenicity as well as receptor binding specificity and affinity ${ }^{24,36,53}$. Changes in affinity or specificity of HA binding can lead to compensatory NA sequence changes ${ }^{54}$. Compensatory NA mutations in egg-passaged viruses predominantly occurred when HA adaptation occurred at the receptorbinding site or surrounding antigenic sites.

Some egg adaptations will lead to detrimental antigenic changes in the HA. Following vaccination with egg-adapted viruses, antibodies may be focussed on the egg-adapted HA sites and not be able to neutralise circulating viruses leading to reduced protection following vaccination with egg-based vaccines. This was demonstrated in this study using a panel of postvaccination (egg-based vaccine) human sera. The sera recognised egg-grown viruses well, but not cell-grown viruses, which more closely resemble circulating influenza viruses. Similar results with human and animal post-vaccination and infection sera has also been demonstrated by others ${ }^{19,38}$. A significant effort is made to minimise the use of virus strains with known egg adaptations in the manufacture of influenza vaccines but this also restricts the number of egg-based CVVs that are available. However, a certain level of adaptation is tolerated as with many viruses some egg adaptive HA and NA changes are inevitable. The level to which this contributes to reduced vaccine efficacy depends on the virus, the subtype, the lineage, and the year in which the egg CVV and the influenza vaccine was produced. To date, this reduced efficacy has been mostly attributed to influenza $A(H 3 N 2)$ viruses ${ }^{25-27,55}$. In 2017/2018 Northern Hemisphere, Flucelvax Quadrivalent was available and contained the $\mathrm{A}(\mathrm{H} 3 \mathrm{~N} 2)$ component that was exclusively derived and manufactured from a cell CVV. Improved effectiveness of this vaccine component as compared to eggpassaged influenza vaccine in preventing influenza-like illness was demonstrated in the $2017 / 2018 \mathrm{NH}$ season, where $\mathrm{A}(\mathrm{H} 3 \mathrm{~N} 2)$ viruses dominated ${ }^{22}$. All four influenza viruses for Flucelvax ${ }^{\circledast}$ Quadrivalent have been isolated and propagated exclusively in MDCK33016PF cells since 2019/2020, and further improvements in vaccine efficacy are expected. There is also a need to develop alternate technologies, such as cell, recombinant or egg-based platforms that do not allow for egg adaptation ${ }^{5,29}$. The potential for 'humanised-eggs', where eggs express the SIAT1 gene are an alternative future approach. The data in this study clearly demonstrate that the successful isolation of human influenza viruses in MDCK33016PF cells offers an alternate, attractive strategy for CVV generation with the potential for improved influenza vaccine protection.

\section{METHODS}

\section{Clinical specimens and viruses}

Human clinical samples that are supplied to the WHO Collaborating Centre for Reference and Research on Influenza in Melbourne (the Centre) fall under the terms of reference of the WHO Global Influenza Surveillance and
Response System (GISRS, see ref. ${ }^{56}$ ) and the use of these samples for influenza vaccine development is permitted without additional ethics approval being held by the Centre. Human seasonal influenza-positive OCS and virus isolates received from 2008-2020 as part of routine influenza surveillance activities from WHO National Influenza Centres, WHO Collaborating Centres and other regional laboratories and hospitals in Australia, New Zealand and Asia/Pacific region, were included in this study. OCS were predominantly throat swabs, nasal swabs and nasopharyngeal swabs as well as a small number of nasopharyngeal aspirates with very few bronchoalveolar lavages. The clinical samples used in this study were chosen to ensure a broad representation of the viral genetic clades present in each type/subtype circulating at the time.

\section{Virus infection and passaging in embryonated hen's eggs}

OCS were diluted $1: 2(\mathrm{v} / \mathrm{v}$ ) in a $1000 \mathrm{U} / \mathrm{ml}$ neomycin sulfate (Pharmacia \& Upjohn) $/ 2000 \mathrm{U} / \mathrm{ml}$ polymyxin B sulfate (Xella Pharmaceutical) solution and $200 \mu \mathrm{l}$ was inoculated into either the amniotic cavity of 13-15 day old embryonated hen's eggs or the allantoic cavity of 10-12 day old embryonated hen's eggs (kindly provided by Seqirus). Inoculated eggs were incubated in a humidified incubator at $35^{\circ} \mathrm{C}$ for $3-4$ days for influenza A viruses and $33^{\circ} \mathrm{C}$ for $3-4$ days for influenza B viruses. Amniotic or allantoic fluid was harvested and the presence of virus was assessed by Haemagglutination (HA) Assay using 1\% turkey or GP RBC. Viruses were passaged up to seven times in eggs, with up to three (blind) passages in the amniotic cavity and up to four passages in the allantoic cavity. For passage, the inoculum was prepared at three different dilutions, into 3-6 eggs per dilution, according to the $\mathrm{HA}$ titre of the prior passage (4-16 HA- diluted $10^{-1}, 10^{-3}, 10^{-5}, 32-512 \mathrm{HA}$-diluted $10^{-2}, 10^{-4}$, $10^{-6},>512 \mathrm{HA}$-diluted $10^{-4}, 10^{-6}, 10^{-8}$ prior to infection).

\section{Passaging of MDCK33016PF cells}

MDCK33016PF suspension cells (a proprietary cell line developed by Novartis ${ }^{31-33}$ and now owned by Seqirus Limited) were maintained at densities between $1-1.5 \times 10^{6} \mathrm{cell} / \mathrm{s} / \mathrm{ml}$ in $500 \mathrm{ml}$ disposable spinner flasks (Corning, USA) in MDCK $33016 \mathrm{CDM}$ (Lonza, Germany) at $37^{\circ} \mathrm{C}, 4.5 \% \mathrm{CO}_{2}$, shaking at $1 \mathrm{~g}$. Cells were passaged at 3-4 day intervals.

\section{Virus infection in MDCK33016PF cells}

Virus infections in cells were performed in a $5 \mathrm{ml}$ total volume in $50 \mathrm{ml}$ filter tubes (TPP, Transadingen, Switzerland), containing $1 \times 10^{6} \mathrm{cell} / \mathrm{s} / \mathrm{ml}$ (range 9.9-1.1 $\left.\times 10^{6}\right)$ in a $(7: 3 \mathrm{v} / \mathrm{v})$ mixture of PFM:CDM infection media (PFM manufactured by Invitrogen, USA, CDM by Lonza, Germany) supplemented with trypsin (1\%, Roche, USA) and neomycin $(37.5 \mu \mathrm{g} / \mathrm{ml}$, Sigma, USA). About $50 \mu \mathrm{l}$ OCS (neat) was inoculated for passage 1 (P1) inoculation, resulting in a $10^{-2}$ dilution. If less than $50 \mu$ OCS was available, the specimen tube was washed with $50 \mu \mathrm{l}$ PBS and this was inoculated into the cells. The virus cell suspension was cultured at $34.5^{\circ} \mathrm{C}, 5 \% \mathrm{CO}_{2}$, shaking at $4 \mathrm{~g}$ for $72 \pm 2 \mathrm{~h}$. To harvest the virus, samples were centrifuged at $500 \times \mathrm{g}$ for $10 \mathrm{~min}$, and the supernatant was collected. The presence of the virus was assessed by HA Assay and flow cytometry (see below). A second passage (P2) of $100 \mu \mathrm{l}$ P1 virus into $10 \mathrm{ml}$ cells was performed for all samples, as above. The $\mathrm{P} 1$ virus inoculum was diluted based on the HA titre (0-8 HA-diluted 1/10; >8-64 HA-diluted $1 / 100$; >64-512 HA-dilution $1 / 1000 ;>512 \mathrm{HA}$-dilution of $1 / 10000$ ). All samples were stored at $-80^{\circ} \mathrm{C}$ unless immediately passaged or analysed. All analyses were performed on P2 virus samples.

\section{Assessment of virus samples by HA Assay and flow cytometry}

Egg- and cell-grown virus isolates were assessed for virus growth by $\mathrm{HA}$ assay. Briefly, 1\% haematocrit turkey or GP RBC was added to an equal volume of virus that had been serially diluted twofold in a microtitre plate ${ }^{3}$.

Virus-infected cells (a total of $10^{4}$ cells), at $72 \mathrm{~h}$ post-inoculation, were fixed and permeabilised using the Cytofix/Cytoperm kit (BD, USA) as per the manufacturer's instructions. Expression of influenza $A$ and $B$ nucleoprotein (NP) was detected using the IMAGEN influenza $A$ and $B$ kit (Cat \# K610511-2, Thermo Scientific, USA), diluted 1:100. Cells were analysed using a FACsCanto II (BD, USA) with FACSDiva ${ }^{\mathrm{TM}}$ software (v9, USA). Single cells were identified by forward and side scatter and the proportion influenza-positive was determined by assessment of fluorescence as compared to a 'mock-infected' cell population. Isolates were classified as positive for growth in cells or eggs if a HA titre of at least 2 was observed for either turkey or GP RBC. Isolates were also classified as 
positive for growth in cells if at least $20 \%$ of cells were positive for NP by flow cytometry in the absence or presence of a detectable HA titre. There was a high positive correlation between flow cytometry results and HA titres for all subtypes/lineages (see Supplementary Information, Supplementary Fig. 2).

\section{Sequencing of HA genes}

RNA was extracted from $140 \mu$ virus isolate or OCS using QIAmp Viral RNA mini kit (Qiagen, Germany) according to the manufacturer's instruction. A $5 \mathrm{ul}$ aliquot of RNA was used to amplify the HA-1 domain of influenza HA using SuperScript III One-Step RT-PCR with Platinum Taq (Invitrogen, USA) with $\mathrm{M} 13$ tagged-gene-specific primers ${ }^{57}$. Unincorporated primers and dNTPs were removed using ExoSAP-IT (Affymetrix, USA), according to the manufacturer's instructions. DNA sequencing was performed with M13 primers (M13F-59 TGTAAAACGACGGCCAGT and M13R 59 CAGGAAACAG (TATGACC) in a 96-well plate format using the BigDye Terminator v3.1 Cycle Sequencing Kit (Thermo Scientific, USA), followed by the removal of excess dye terminators with a BigDye XTerminator purification kit (Thermo Scientific, USA). The sequence was determined using an automated capillary DNA sequencer (ABI Prism 3500xL). Identification of mixed bases was set at $25 \%$. Sequences were assembled as consensus using DNASTAR Lasergene Suite v.9.1.0, Seqman v9.1 and compared using Megalign (v9.1).

\section{Haemagglutination inhibition assay}

Pre- and post-vaccination sera were used to analyse antibody responses. Subjects were enroled in a clinical trial at the Royal Adelaide Women and Children's hospital, and blood was drawn prior to influenza vaccination and 21 days post-vaccination. Subjects were vaccinated with the Seqirus Southern Hemisphere quadrivalent vaccine for the 2016 season (the vaccine contained an A/California/7/2009-like virus, an A/Hong Kong/4801/ 2014-like virus and a B/Brisbane/60/2008-like virus). Sera was sent to the WHO CC in Melbourne, and antibody responses to the four vaccine components were assessed using the Haemagglutination inhibition (HI) assay using both egg and cell-derived viruses ${ }^{3}$. Serum samples were pretreated with receptor destroying enzyme II (Denka Seiken Co. Ltd.), 1:5 (volume/volume). Influenza virus (4 HA units, $25 \mu \mathrm{l}$ ) was added to an equal volume of treated serum that had been serially diluted twofold in a microtitre plate. Following a 1-h incubation, $25 \mu \mathrm{l} 1 \%$ (volume/volume) guinea pig RBC was added to each well. HI was read after $45 \mathrm{~min}$. Titres were expressed as the reciprocal of the highest dilution of serum where Haemagglutination was inhibited.

\section{Statistical analysis}

The proportion of viruses isolated in eggs as compared to cells was assessed using Chi-squared analysis. The difference in variation in isolation rates over the years studied between egg and cells was assessed using the $F$-test, calculated in Excel (Microsoft, Australia). The difference in HA titres or Cts between viruses isolated only in cells as compared to viruses isolated in both cells and eggs was assessed using an unpaired $t$-test, using GraphPad Prism Version 7. The difference in $\mathrm{HI}$ titres between cell and egggrown isolates was assessed using a multiple $t$-test, using GraphPad Prism Version 7.

\section{Reporting Summary}

Further information on research design is available in the Nature Research Reporting Summary linked to this article.

\section{DATA AVAILABILITY}

The datasets generated during and/or analysed during the current study are available from the corresponding author on reasonable request.

Received: 27 May 2021; Accepted: 12 November 2021; Published online: 09 December 2021

\section{REFERENCES}

1. Paget, J. et al. Global mortality associated with seasonal influenza epidemics: new burden estimates and predictors from the GLaMOR Project. J. Glob. Health 9, 020421 (2019).
2. Iuliano, A. D. et al. Estimates of global seasonal influenza-associated respiratory mortality: a modelling study. Lancet 391, 1285-1300 (2018).

3. WHO. Manual for the Laboratory Diagnosis and Virological Surveillance of Influenza (WHO, 2011).

4. Baez, M., Palese, P. \& Kilbourne, E. D. Gene composition of high-yielding influenza vaccine strains obtained by recombination. J. Infect. Dis. 141, 362-365 (1980).

5. Rockman, S., Laurie, K. L., Parkes, S., Wheatley, A. \& Barr, I. G. New technologies for influenza vaccines. Microorganisms 8, 1745 (2020).

6. Merten, O. W., Hannoun, C., Manuguerra, J. C., Ventre, F. \& Petres, S. Production of influenza virus in cell cultures for vaccine preparation. Adv. Exp. Med. Biol. 397, 141-151 (1996).

7. Schepetiuk, S. K. \& Kok, T. The use of MDCK, MEK and LLCMK2 cell lines with enzyme immunoassay for the isolation of influenza and parainfluenza viruses from clinical specimens. J. Virol. Methods 42, 241-250 (1993).

8. Gaush, C. R. \& S, T. F. Replication and plaque assay of influenza virus in an established line of canine kidney cells. Appl. Microbiol. 16, 588-594 (1968).

9. Oh, D. Y., Barr, I. G., Mosse, J. A. \& Laurie, K. L. MDCK-SIAT1 cells show improved isolation rates for recent human influenza viruses compared to conventional MDCK cells. J. Clin. Microbiol. 46, 2189-2194 (2008).

10. Lee, J. M. et al. Deep mutational scanning of hemagglutinin helps predict evolutionary fates of human H3N2 influenza variants. Proc. Natl Acad. Sci. USA 115, E8276-E8285 (2018)

11. Takada, K. et al. A humanized MDCK cell line for the efficient isolation and propagation of human influenza viruses. Nat. Microbiol. 4, 1268-1273 (2019).

12. Govorkova, E. A., Kaverin, N. V., Gubareva, L. V., Meignier, B. \& Webster, R. G. Replication of influenza $A$ viruses in a green monkey kidney continuous cell line (Vero). J. Infect. Dis. 172, 250-253 (1995).

13. Govorkova, E. A., Murti, G., Meignier, B., de Taisne, C. \& Webster, R. G. African green monkey kidney (Vero) cells provide an alternative host cell system for influenza A and B viruses. J. Virol. 70, 5519-5524 (1996).

14. Seo, S. H., Goloubeva, O., Webby, R. \& Webster, R. G. Chacterization of a porcine lung epithelial cell line suitable for influenza virus studies. J. Virol. 75, 9517-9525 (2001).

15. Meliopoulos, V. et al. Primary swine respiratory epithelial cell lines for the efficient isolation and propagation of influenza A viruses. J. Virol. 94, e01091-20. (2020).

16. Brown, S. W. \& Mehtali, M. The avian EB66 cell line, application to vaccines, and therapeutic protein production. PDA J. Pharm. Sci. Tech. 64, 419-425 (2010).

17. Girard, M. P., Tam, J. S., Assossou, O. M. \& Kieny, M. P. The 2009 A (H1N1) influenza virus pandemic: a review. Vaccine 28, 4895-4902 (2010).

18. Rockman, S., Laurie, K. L. \& Barr, I. Pandemic influenza vaccines:what did we learn from the 2009 pandemic and are we better prepared now? Vaccines 8, 211 (2020).

19. Zost, S. J. et al. Contemporary H3N2 influenza viruses have a glycosylation site that alters binding of antibodies elicited by egg-adapted vaccine strains. Proc. Natl Acad. Sci. USA 114, 12578-83. (2017).

20. Park, Y. W. et al. Comparison of antigenic mutation during egg and cell passage cultivation of H3N2 influenza virus. Clin. Exp. Vaccin. Res. 9, 56-63 (2020).

21. Meyer, W. J. et al. Influence of host cell-mediated variation on the international surveillance of influenza A (H3N2) viruses. Virology 196, 130-137 (1993).

22. Barr, I. G. et al. Cell culture-derived influenza vaccines in the severe 2017-2018 epidemic season: a step towards improved influenza vaccine effectiveness. $n p j$ Vaccines 3, 44 (2018).

23. Saito, T. et al. Antigenic alteration of influenza B virus associated with loss of a glycosylation site due to host-cell adaptation. J. Med. Virol. 74, 336-343 (2004).

24. Parker, L. et al. Effects of egg-adaptation on receptor-binding and antigenic properties of recent influenza A (H3N2) vaccine viruses. J. Gen. Virol. 97, 1333-44. (2016).

25. Barr, I. G. et al. WHO recommendations for the viruses used in the 2013-2014 Northern Hemisphere influenza vaccine: epidemiology, antigenic and genetic characteristics of influenza $A(\mathrm{H} 1 \mathrm{~N} 1)$ pdm09, $A(\mathrm{H} 3 \mathrm{~N} 2)$ and $B$ influenza viruses collected from October 2012 to January 2013. Vaccine 32, 4713-4725 (2014).

26. Skowronski, D. M. et al. Low 2012-13 influenza vaccine effectiveness associated with mutation in the egg-adapted H3N2 vaccine strain not antigenic drift in circulating viruses. PLOS ONE 9, e92153 (2014).

27. Budd, A. P. et al. Update: influenza activity - United States, October 1, 2017-February 3, 2018. MMWR Morb. Mortal. Wkly. Rep. 67, 169-179 (2018).

28. WHO. WHO recommendations on the composition of influenza virus vaccines. https://www.who.int/influenza/vaccines/virus/recommendations/en/ (2020).

29. Wu, N. C. et al. Preventing an antigenically disruptive mutation in egg-based $\mathrm{H} 3 \mathrm{~N} 2$ seasonal influenza vaccines by mutational incompatibility. Cell Host Microbe 25, 836-44. (2019).

30. Onions, D., Egan, W., Jarrett, R., N, R. \& Gregersen, J. P. Validation of the safety of MDCK cells as a substrate for the production of a cell-derived influenza vaccine. Biologicals 38, 544-551 (2010). 
31. Roth, B., Mohr, H., Enders, M., Garten, W. \& Gregersen, J.-P. Isolation of influenza viruses in MDCK 33016PF cells and clearance of contaminating respiratory viruses. Vaccine 30, 517-522 (2012).

32. Gregersen, J. P. A risk-assessment model to rate the occurrence and relevance of adventitious agents in the production of influenza vaccines. Vaccine $\mathbf{2 6}$, 3297-3304 (2008).

33. Gregersen, J. P. A quantitative risk assessment of exposure to adventitious agents in a cell culture-derived subunit influenza vaccine. Vaccine 26, 3332-40. (2008)

34. Jorquera, P. A. et al. Insights into the antigenic advancement of influenza A (H3N2) viruses, 2011-2018. Sci. Rep. 9, 2676 (2019).

35. Ovsyannikova, I. G., White, S. J., Albrecht, R. A., G-S, A. \& Poland, G. A. Turkey versus guinea pig red blood cells: hemagglutination differences alter hemagglutination inhibition responses against influenza A/H1N1. Viral. Immunol. 27, 174-178 (2014).

36. Daniels, R. S. et al. Antigenic analyses of influenza virus haemagglutinins with different receptor-binding specificities. Virol. 138, 174-177 (1984).

37. Lu, B., Zhou, H., Chan, W., Kemble, G. \& Jin, H. Single amino acid substitutions in the hemagglutinin of influenza A/Singapore/21/04 (H3N2) increase virus growth in embryonated chicken eggs. Vaccine 24, 6691-6693 (2006).

38. Gouma, S., W, M. \& Hensley, S. E. Antigenic assessment of the H3N2 component of the 2019-2020 Northern Hemisphere influenza vaccine. Nat. Commun. 11, 2445 (2020).

39. Belongia, E. A. \& McLean, H. Q. Influenza vaccine effectiveness: defining the H3N2 problem. Clin. Infect. Dis. 69, 1817-23. (2019).

40. Wen, F., Huang, S. \& Guo, J. Egg adaptive mutation patterns of H3N2 human influenza A viruses. J. Infect. 80, 232-54. (2020).

41. Byrd-Leotis, L. et al. Influenza binds phosphorylated glycans from human lung. Sci. Adv. 5, eaav2554 (2019).

42. Matrosovich, M. N., Matrosovich, T. Y., Gray, T., Roberts, N. A. \& Klenk, H. D. Human and avian influenza viruses target different cell types in cultures of human airway epithelium. Proc. Natl Acad. Sci. USA 101, 4620-4624 (2004).

43. Thompson, C. I., Barclay, W. S., Zambon, M. C. \& Pickles, R. J. Infection of human airway epithelium by human and avian strains of influenza $A$ virus. J. Virol. 80 , 8060-8068 (2006).

44. Walther, T. et al. Glycomic analysis of human respiratory tract tissues and correlation with influenza virus infection. PLoS Pathog. 9, e1003223 (2013).

45. Matrosovich, M., Matrosovich, T., Carr, J., Roberts, N. A. \& Klenk, H.-D. Overexpression of the a-2,6-sialyltransferase in MDCK cells increases influenza virus sensitivity to neuraminidase inhibitors. J. Virol. 77, 8418-25. (2003).

46. Kuchipudi, S. V. et al. Differences in influenza virus receptors in chickens and ducks: implications for interspecies transmission. J. Mol. Genet. Med. 3, 143-151 (2009).

47. Pillai, S. P. \& L, C. Species and age related differences in the type and distribution of influenza virus receptors in different tissues of chickens, ducks and turkeys. Virol. J. 7, 5 (2010).

48. Sriwilaijaroen, N. et al. Analysis of N-glycans in embryonated chicken egg chorioallantoic and amniotic cells responsible for binding and adaptation of human and avian influenza viruses. Glycoconj. J. 26, 433-443 (2009).

49. Lin, Y. P. et al. Evolution of the receptor binding properties of the influenza A (H3N2) hemagglutinin. Proc. Natl Acad. Sci. USA 109, 21474-21479 (2012).

50. Lin, Y. P. et al. Neuraminidase receptor binding variants of human influenza $A$ (H3N2) viruses resulting from substitution of aspartic acid 151 in the catalytic siteL a role in virus attachment? J. Virol. 84, 6769-6781 (2010).

51. Lin, Y. et al. The characteristics and antigenic properties of recently emerged subclade 3 C.3a and 3C.2a human influenza $\mathrm{A}(\mathrm{H} 3 \mathrm{~N} 2)$ viruses passaged in MDCK cells. Influenza Other Respir. Viruses 11, 263-74. (2017).

52. Drake, J. W. Rates of spontaneous mutation among RNA viruses. Proc. Natl Acad. Sci. USA 90, 4171-4175 (1993).

53. Hensley, S. E. et al. Hemagglutinin receptor binding avidity drives influenza $A$ virus antigenic drift. Science 326, 734-736 (2009).

54. Mitnaul, L. J. et al. Balanced hemagglutinin and neuraminidase activities are critical for efficient replication of influenza A virus. J. Virol. 74, 6015-6020 (2000).

55. Sullivan, S. G. et al. Low interim influenza vaccine effectiveness, Australia, 1 May to 24 September 2017. Eur. Surveill. 22, 17-00707. (2017)
56. WHO. WHO collaborating centres for influenza and their terms of reference. https://www.who.int/influenza/gisrs laboratory/collaborating_centres/list/en/ (2021)

57. Deng, Y.-M. et al. A simplified Sanger sequencing method for full genome sequencing of multiple subtypes of human influenza A viruses. J. Clin. Virol. 68 43-48 (2015).

58. Bll Flusurver. http://flusurver.bii.a-star.edu.sg (2020).

59. Sun, W. et al. Antibody responses toward the major antigenic sites of influenza $B$ virus hemagglutinin in mice, ferrets, and humans. J. Virol. 93, e01673-18. (2019).

60. Wang, Q., Cheng, F., Lu, M., Tian, X. \& Ma, J. Crystal structure of unligated influenza B virus hemagglutinin. J. Virol. 82, 3011-3020 (2008).

\section{ACKNOWLEDGEMENTS}

The Melbourne WHO Collaborating Centre for Reference and Research on Influenza is supported by the Australian Government Department of Health. The authors acknowledge Simone Blayer, Ted Tsai, Jeff Pavlicek, Avishek Nandi, Chris Gully, Joelle Dharmakumara, Scott Reddiex, Jayde Galetti, Danielle Tilmanis and Robert Shaw for their contribution to the project.

\section{AUTHOR CONTRIBUTIONS}

I.G.B. and H.T. conceived and conceptualised the work. H.P., H.L., S.S., C.R. and C.B. performed experiments. K.L.L., H.P., V.L., S.R. and I.G.B. analysed and interpreted data. K.L.L., H.P., S.R. and I.G.B. wrote the manuscript. H.P. and K.L.L. are considered the cofirst author. All authors supported the review of the manuscript.

\section{COMPETING INTERESTS}

K.L.L., C.B. and S.R. are employees of Seqirus Ltd that manufactures influenza seasonal and pandemic vaccines. I.G.B. has shares in an influenza vaccine manufacturing company.

\section{ADDITIONAL INFORMATION}

Supplementary information The online version contains supplementary material available at https://doi.org/10.1038/s41541-021-00415-3.

Correspondence and requests for materials should be addressed to Heidi Peck or Karen L. Laurie.

Reprints and permission information is available at http://www.nature.com/ reprints

Publisher's note Springer Nature remains neutral with regard to jurisdictional claims in published maps and institutional affiliations.

Open Access This article is licensed under a Creative Commons Attribution 4.0 International License, which permits use, sharing, adaptation, distribution and reproduction in any medium or format, as long as you give appropriate credit to the original author(s) and the source, provide a link to the Creative Commons license, and indicate if changes were made. The images or other third party material in this article are included in the article's Creative Commons license, unless indicated otherwise in a credit line to the material. If material is not included in the article's Creative Commons license and your intended use is not permitted by statutory regulation or exceeds the permitted use, you will need to obtain permission directly from the copyright holder. To view a copy of this license, visit http://creativecommons. org/licenses/by/4.0/.

(c) The Author(s) 2021 